\title{
Three distinct recovery patterns following primary total knee arthroplasty: dutch arthroplasty register study of 809 patients
}

\author{
Jeroen C. van Egmond ${ }^{1}$ - Brechtje Hesseling ${ }^{1} \cdot$ Marijke Melles $^{2,3}$. Stephan B. W. Vehmeijer ${ }^{1}$. \\ Liza N. van Steenbergen ${ }^{4} \cdot$ Nina M. C. Mathijssen ${ }^{1} \cdot$ Jarry T. Porsius ${ }^{5,6}$
}

Received: 22 December 2019 / Accepted: 24 March 2020

(c) European Society of Sports Traumatology, Knee Surgery, Arthroscopy (ESSKA) 2020

\begin{abstract}
Purpose Total knee arthroplasty (TKA) is usually effective, although not all patients have satisfactory outcomes. This assumes distinct recovery patterns might exist. Little attention has been paid to determine which patients have worse outcomes. This study attempts to distinguish specific recovery patterns using the Oxford knee score (OKS) during the first postoperative year. The secondary aim was to explore predictors of less favourable recovery patterns.

Methods Analysis of patients in the Dutch Arthroplasty Register (LROI) with unilateral primary TKA. Data collected up to one year postoperative was used. To identify subgroups of patients based on OKS, latent class growth modeling (LCGM) was used. Moreover, multivariable multinomial logistic regression analysis was used to explore predictors of class membership. Results 809 Patients completed three OKS during the first year postoperative and were included. LCGM identified 3 groups of patients; 'high risers' (most improvement during first 6-months, good 12-month scores 77\%), 'gradual progressors' (continuous improvement during the first year 13\%) and 'non responders' (initial improvement and subsequent deterioration to baseline score 10\%). Predictors of least favourable class membership (OR, 95\%CI) are EQ-5D items: VAS health score $(0.83,0.73-0.95)$, selfcare $(2.22,1.09-4.54)$ and anxiety/depression $(2.45,1.33-4.52)$.

Conclusion Three recovery patterns after TKA were distinguished; 'high risers', 'gradual progressors' and 'non responders'. Worse score on EQ-5D items VAS health, selfcare, and anxiety/depression were correlated with the least favourable 'non responders' recovery pattern.
\end{abstract}

Keywords Total knee arthroplasty $\cdot$ Latent class growth modeling $\cdot$ Trajectories $\cdot$ Patient-reported outcome measurements

Electronic supplementary material The online version of this article (https://doi.org/10.1007/s00167-020-05969-8) contains supplementary material, which is available to authorized users.

Jeroen C. van Egmond

j.vanegmond@rdgg.nl

1 Department of Orthopaedic Surgery, Reinier de Graaf Groep, Reinier de Graafweg 5, 2625 AD Delft, The Netherlands

2 Faculty of Industrial Design Engineering, Delft University of Technology, Landbergstraat 15, 2628 CE Delft, The Netherlands

3 Department of Public and Occupational Health, Amsterdam Public Health Research Institute, Amsterdam UMC, Vrije Universiteit Amsterdam, Van der Boechorststraat 7, 1081 BT Amsterdam, The Netherlands

\section{Introduction}

Approximately $20 \%$ of patients reported being dissatisfied with the results of total knee arthroplasty (TKA) [2, 4]. To improve preoperative consultation and postoperative

4 Dutch Arthroplasty Register (Landelijke Registratie Orthopedische Implantaten/LROI), Bruistensingel 230, 5232 AD Hertogenbosch, The Netherlands

5 Integrated Brain Health Clinical and Research Program, Department of Psychiatry, Massachusetts General Hospital, Harvard Medical School, One Bowdoin Square, Boston, MA 02114, USA

6 Department of Rehabilitation Medicine and the Department of Plastic and Reconstructive Surgery, Erasmus University Medical Center, Dr. Molewaterplein 40, 3015 GD Rotterdam, The Netherlands 
rehabilitation, better understanding of the differences in recovery patterns and the patient characteristics which are associated with pattern membership is needed.

A valuable statistical method to obtain insight into recovery patterns is latent class growth modeling (LCGM) [16]. LCGM is a very suitable method when studying the results of TKA based not only on absolute or relative outcomes, but on the trajectory leading up to these outcomes.

Previous studies using LCGM to analyse pain and function trajectories during the first years after TKA were based on data from single institutions, and demonstrated considerable heterogeneity in recovery after TKA $[6,7,14,17]$.

However, it is still unclear yet whether this heterogeneity is best characterized by two or more than two distinct trajectories. In addition, there is limited information regarding predictors for distinct recovery trajectories. Therefore, trajectories of TKA recovery needs to be investigated in a large nationwide database. The findings will be more generalizable in comparison to single institutional data.

This present study will be the first that investigated TKA recovery trajectories in a large nationwide sample of patients from an arthroplasty registry. The primary objective was to characterize subgroups of patients after TKA according to their Oxford knee score (OKS). The second objective was to determine which patient characteristics were associated with a negative trajectory class membership. The outcome of this study will increase insight into recovery trajectories after primary TKA and might provide indications to further improve and personalize quality of care.

\section{Materials and methods}

Data were retrieved from the Dutch Arthroplasty Register (Landelijke Registratie Orthopedische Implantaten: LROI). The LROI started collecting patient-related outcome measures (PROMS) in 2014 [8]. The registry includes 99\% of all arthroplasties performed in general hospitals, university hospitals and private clinics in the Netherlands [9, 24].

All data obtained from the LROI database were prospectively collected. All patients with primary unilateral TKA for osteoarthritis who were operated between January 2014 and December 2016 were included.

The OKS [5] determined preoperatively, and 6- and 12-months postoperatively were collected. Patients were excluded when the OKS was not completed at all these three time points. Data were also retrieved on the following patient characteristics: age, sex, smoking, American Society of Anaesthesiologists (ASA), Charnley score, body mass index (BMI) and any previous surgery. Previous surgery includes arthroscopy, osteotomy, anterior cruciate ligament repair, meniscectomy, osteosynthesis, patella realignment or synovectomy. Furthermore, the following additional
PROMS were retrieved: Numeric Rating Scale (NRS) for pain, and EuroQol-5D-3L (EQ-5D) [18].

All data were registered as part of routine clinical care, and the present study placed no additional burden on the patient. Therefore, no ethical approval was necessary according to the Dutch Medical Research Involving Human Subjects Act (WMO). All data were handled in line with the Helsinki Declaration.

\section{Statistical analysis}

To clean the data and provide descriptive statistics of the overall sample IBM SPSS version 25 (IBM Corp. Armonk, NY: IBM Corp.) was used. To distinguish trajectories, latent class growth analysis (LCGA) and growth mixture modeling (GMM) analysis were performed in Mplus Version 8.1 (Los Angeles, CA: Muthén \& Muthén). A $p$ value of 0.05 or less was considered statistically significant.

\section{Outcome}

The trajectories were based on reported problems with the operated knee, which were determined by means of the OKS. The OKS is based on 12 questions regarding pain and function of the knee. Total score ranges from 0 to 48 with higher scores indicating better function and less pain [5].

\section{Model selection}

In this present study LCGM was used. One advantage of LCGM lies in the assumption that there are two or more unobserved subgroups with each their own intercept and slope (i.e., starting point and change over time), as opposed to conventional growth modeling which assumes a single population with one intercept and one slope. Another advantage is that it allows each subgroup to demonstrate a unique pattern of change over time; the subgroups do not need to display the same overall shape for recovery pattern.

Within LCGM, LCGA and GMM also differ from each other: where LCGA assumes there is no variability in growth factors within groups, GMM does allow within-group variability in growth factors.

Based on previous studies it was presumed that 2 to 4 classes could be identified $[6,7,14,17]$. Starting with a conventional growth model to assess the overall degree of heterogeneity between patients; in this model the intercept and slope variance was estimated as well as the covariance in the sample as a whole [12]. For a full exploration of distinct trajectories, 1-class to 6-class LCGA and GMM models were fitted and compared to the results of the conventional growth model. In all models, a latent basis model was specified for the growth pattern preventing to force a predefined shape of recovery trajectory, such as a linear shape, onto 
the data $[3,19]$. This also allowed to estimate the amount of change between the preoperative measurement and the 12 months measurement (i.e., the estimated mean slopes in the models), while also estimating how much of that change occurred at 6 months (i.e., the estimated factor loading of the 6 months measurement).

In both the LCGA and GMM models, the pattern of change and the means of the growth factors were estimated per class. The free residual variances were estimated for the overall model only. In the LCGA models, variance and covariance are naturally restricted to zero. In the GMM models, variance and covariance were only estimated for the overall model, not per class.

There are no definitive decision criteria for the optimal number of classes. However, model specification and selection should be guided by theory, previous empirical findings, and initial examinations of the data $[3,19,23]$.

Model selection was based on a combination of indices of fit [19], including the following four indices: (1) visual inspection of the plots and parsimony, interpretability and clinical meaningfulness of the model; (2) the relative fit statistics Bayesian Information Criteria (BIC), Akaike Information Criteria (AIC) and Adjusted BIC, where lower values indicate a better fit; (3) entropy, where higher values indicate a higher confidence in the correct classification of individuals; and (4) the Bootstrapped Likelihood Ratio Test (BLRT). Based on these criteria, a final model was chosen to further explore patient characteristics associated with the different trajectories of recovery. All models were run with 500 random starting values and 20 final iterations, and subsequently rerun with 2000 random starting values and 400 final iterations to ensure that the optimal solution was found. Common procedures were followed to check whether models were local solutions [26].

The r3step procedure in Mplus was used to perform univariable and multivariable multinomial logistic regression analyses, in which the smaller subgroups of patients were compared to the largest group of patients.

\section{Predictors}

After selecting the final model, preoperative patient characteristics across the different classes were compared. Characteristics of interest included age (dichotomized into $\leq 75$ years and $>75$ years), sex, smoking, Charnley score, BMI (normal, overweight and obese), ASA (dichotomized into ASA I-II and III-IV) and previous surgery on the affected joint. Moreover, EQ-5D scores (depression, selfcare, pain, daily activities, and VAS health score) were compared [10]. EQ-5D scores were dichotomized (no problems vs. moderate-to-severe problems) if group sizes became too small for subgroup analysis.

\section{Results}

\section{Patient characteristics}

Complete preoperative OKS as well as 6- and 12-months postoperative OKS were available for 809 patients and were included in the analysis. Table 1 presents all patient characteristics for the whole group as well as for each class in the final model.

\section{Selection of final model}

The conventional one-class growth model showed a large amount of variability in preoperative OKS and longitudinal change. Although the fit statistics of LCGA and GMM models continued to improve up to a 6-class model, this started to flatten out above the 3-class model. (Table 2 ) In addition, group sizes became very small in models with more than 3 -classes, and eventually the additional classes were not considered clinically relevant, since additional groups were slight variations of the 3-class model.

The smaller classes were more heterogeneous in the LCGA models than in the GMM models; because of this heterogeneity and the worse fit statistics of LCGA, was continued with the GMM models.

Based on a parsimonious solution, and on the combination of distinct trajectories, entropy and class sizes, the 3-class GMM model was chosen as final model (Fig. 1). In all models, the largest class was the most homogeneous and the other classes were more heterogeneous (Fig. 2). This 3 -class model had an entropy of 0.868 , and the average posterior class probability of all classes was above 0.70 , which indicates good class separation (see appendix for additional information) $[15,19]$.

The largest group was labelled as 'high risers', since this group continued to improve after most of the improvement on OKS was obtained during the first six months. The middle group was labelled as 'gradual progressors' due to the subsequent improvement on OKS after a medium improvement during the first six months. The smallest group was labelled as 'non responders', since this patient group showed a deterioration in OKS after an initial improvement during the first six months after surgery. Table 3 presents preoperative and 6-month and 12-month postoperative OKS for each class.

\section{Univariable analysis}

For the univariable analysis, the largest group 'high risers' was chosen as the reference group. The following variables were significant (OR, 95\% CI) for class membership of 'non 
Table 1 Descriptive of the entire sample and of the three separated trajectories

\begin{tabular}{|c|c|c|c|c|}
\hline & $\begin{array}{l}\text { Entire sample } \\
(n=809)\end{array}$ & $\begin{array}{l}\text { High risers } \\
(n=623,77 \%)\end{array}$ & $\begin{array}{l}\text { Gradual progressors } \\
(n=108,13.4 \%)\end{array}$ & $\begin{array}{l}\text { Non responders } \\
(n=78,9.6 \%)\end{array}$ \\
\hline Age, mean (SD) years $[95 \% \mathrm{CI}]$ & $\begin{array}{l}67.2(8.1) \\
{[66.7-67.8]}\end{array}$ & $\begin{array}{l}67.3(7.8) \\
{[66.7-67.9]}\end{array}$ & $\begin{array}{l}66.6(9.1) \\
{[64.9-68.4]}\end{array}$ & $\begin{array}{l}67.5(9.1) \\
{[65.4-69.5]}\end{array}$ \\
\hline \multicolumn{5}{|l|}{$\operatorname{Sex}, n(\%)$} \\
\hline Male & $296(37 \%)$ & $230(37 \%)$ & $43(40 \%)$ & $23(30 \%)$ \\
\hline Female & $513(63 \%)$ & $393(63 \%)$ & $65(60 \%)$ & $55(70 \%)$ \\
\hline \multicolumn{5}{|l|}{ Smoking, $n(\%)$} \\
\hline No & $741(93 \%)$ & $575(94 \%)$ & $95(89 \%)$ & $71(93 \%)$ \\
\hline Yes & $54(7 \%)$ & $37(6 \%)$ & $12(11 \%)$ & $5(7 \%)$ \\
\hline \multicolumn{5}{|l|}{ ASA score, $n(\%)$} \\
\hline I-II & $674(83 \%)$ & $530(85 \%)$ & $89(82 \%)$ & $55(70 \%)$ \\
\hline III-IV & $134(17 \%)$ & $92(15 \%)$ & $19(18 \%)$ & $23(30 \%)$ \\
\hline \multicolumn{5}{|l|}{$B M I, n(\%)$} \\
\hline Normal weight (BMI 20-25) & $143(18 \%)$ & $118(19 \%)$ & $16(15 \%)$ & $9(12 \%)$ \\
\hline Overweight (BMI 25-30) & $354(44 \%)$ & $277(45 \%)$ & $45(42 \%)$ & $32(41 \%)$ \\
\hline Obese $(\mathrm{BMI}>30)$ & $306(38 \%)$ & $223(36 \%)$ & $47(43 \%)$ & $36(47 \%)$ \\
\hline \multicolumn{5}{|l|}{ Previous surgery on affected knee, $n(\%)$} \\
\hline No & $524(66 \%)$ & $399(65 \%)$ & $73(69 \%)$ & $52(67 \%)$ \\
\hline Yes & $274(34 \%)$ & $215(35 \%)$ & $33(31 \%)$ & $26(33 \%)$ \\
\hline \multicolumn{5}{|l|}{ Charnley score, $n(\%)$} \\
\hline B1 & $397(49 \%)$ & $308(49 \%)$ & $52(48 \%)$ & $37(48 \%)$ \\
\hline B1 & $234(29 \%)$ & $184(30 \%)$ & $32(30 \%)$ & $18(23 \%)$ \\
\hline B2 & $150(19 \%)$ & $111(18 \%)$ & $20(19 \%)$ & $19(25 \%)$ \\
\hline $\mathrm{C}$ & $25(3 \%)$ & $18(3 \%)$ & $4(4 \%)$ & $3(4 \%)$ \\
\hline Pain at rest, mean (SD) $[95 \% \mathrm{CI}]$ & $\begin{array}{l}5.49(2.42) \\
{[5.27-5.70]}\end{array}$ & $\begin{array}{l}5.28(2.42) \\
{[5.03-5.52]}\end{array}$ & $\begin{array}{l}6.02(2.32) \\
{[5.44-6.59]}\end{array}$ & $\begin{array}{l}6.29(2.36) \\
{[5.65-6.92]}\end{array}$ \\
\hline Pain during activity, mean (SD) $[95 \% \mathrm{CI}]$ & $\begin{array}{l}7.54(1.72) \\
{[7.39-7.70]}\end{array}$ & $\begin{array}{l}7.40(1.77) \\
{[7.22-7.58]}\end{array}$ & $\begin{array}{l}7.79(1.66) \\
{[7.38-8.20]}\end{array}$ & $\begin{array}{l}8.20(1.12) \\
{[7.90-8.50]}\end{array}$ \\
\hline \multicolumn{5}{|l|}{ EQ-5D item 'Mobility', $n(\%)$} \\
\hline No problems & $47(5 \%)$ & $40(6 \%)$ & $4(4 \%)$ & $3(4 \%)$ \\
\hline Some problems in walking about & $754(94 \%)$ & $575(93 \%)$ & $104(96 \%)$ & $75(96 \%)$ \\
\hline Confined to bed & $3(1 \%)$ & $3(1 \%)$ & $0(0 \%)$ & $0(0 \%)$ \\
\hline \multicolumn{5}{|l|}{ EQ-5D item 'Self-Care', $n(\%)$} \\
\hline No problems & $692(86 \%)$ & $548(88 \%)$ & $89(83 \%)$ & $55(71 \%)$ \\
\hline Some problems washing or dressing & $108(13 \%)$ & $68(11 \%)$ & $18(17 \%)$ & $22(28 \%)$ \\
\hline Unable to wash or dress & $4(1 \%)$ & $3(1 \%)$ & $0(0 \%)$ & $1(1 \%)$ \\
\hline \multicolumn{5}{|l|}{ EQ-5D item 'Usual Activities', $n(\%)$} \\
\hline No problems & $136(17 \%)$ & $109(18 \%)$ & $17(16 \%)$ & $10(13 \%)$ \\
\hline Some problems performing usual activities & $628(78 \%)$ & $489(79 \%)$ & $84(78 \%)$ & $55(70 \%)$ \\
\hline Unable to perform usual activities & $41(5 \%)$ & $22(3 \%)$ & $6(6 \%)$ & $13(17 \%)$ \\
\hline \multicolumn{5}{|l|}{ EQ-5D item 'Pain/Discomfort', $n(\%)$} \\
\hline No pain or discomfort & $56(7 \%)$ & $45(7 \%)$ & $6(5 \%)$ & $5(6 \%)$ \\
\hline Moderate pain or discomfort & $568(71 \%)$ & $452(73 \%)$ & $77(72 \%)$ & $39(50 \%)$ \\
\hline Extreme pain or discomfort & $181(22 \%)$ & $123(20 \%)$ & $24(23 \%)$ & $34(44 \%)$ \\
\hline \multicolumn{5}{|l|}{ EQ-5D item 'Anxiety/Depression', $n(\%)$} \\
\hline Not anxious or depressed & $638(79 \%)$ & $509(82 \%)$ & $82(76 \%)$ & $47(60 \%)$ \\
\hline Moderate anxious or depressed & $146(18 \%)$ & $97(16 \%)$ & $22(20 \%)$ & $27(35 \%)$ \\
\hline Extremely anxious or depressed & $22(3 \%)$ & $14(2 \%)$ & $4(4 \%)$ & $4(5 \%)$ \\
\hline EQ-5D VAS health score, mean (SD) $[95 \% \mathrm{CI}]$ & $\begin{array}{l}70.07(17.77) \\
{[68.83-71.32]}\end{array}$ & $\begin{array}{l}71.54(17.52) \\
{[70.14-72.95]}\end{array}$ & $\begin{array}{l}66.84(17.66) \\
{[63.340-70.27]}\end{array}$ & $\begin{array}{l}62.89(17.77) \\
{[58.83-66.96]}\end{array}$ \\
\hline
\end{tabular}




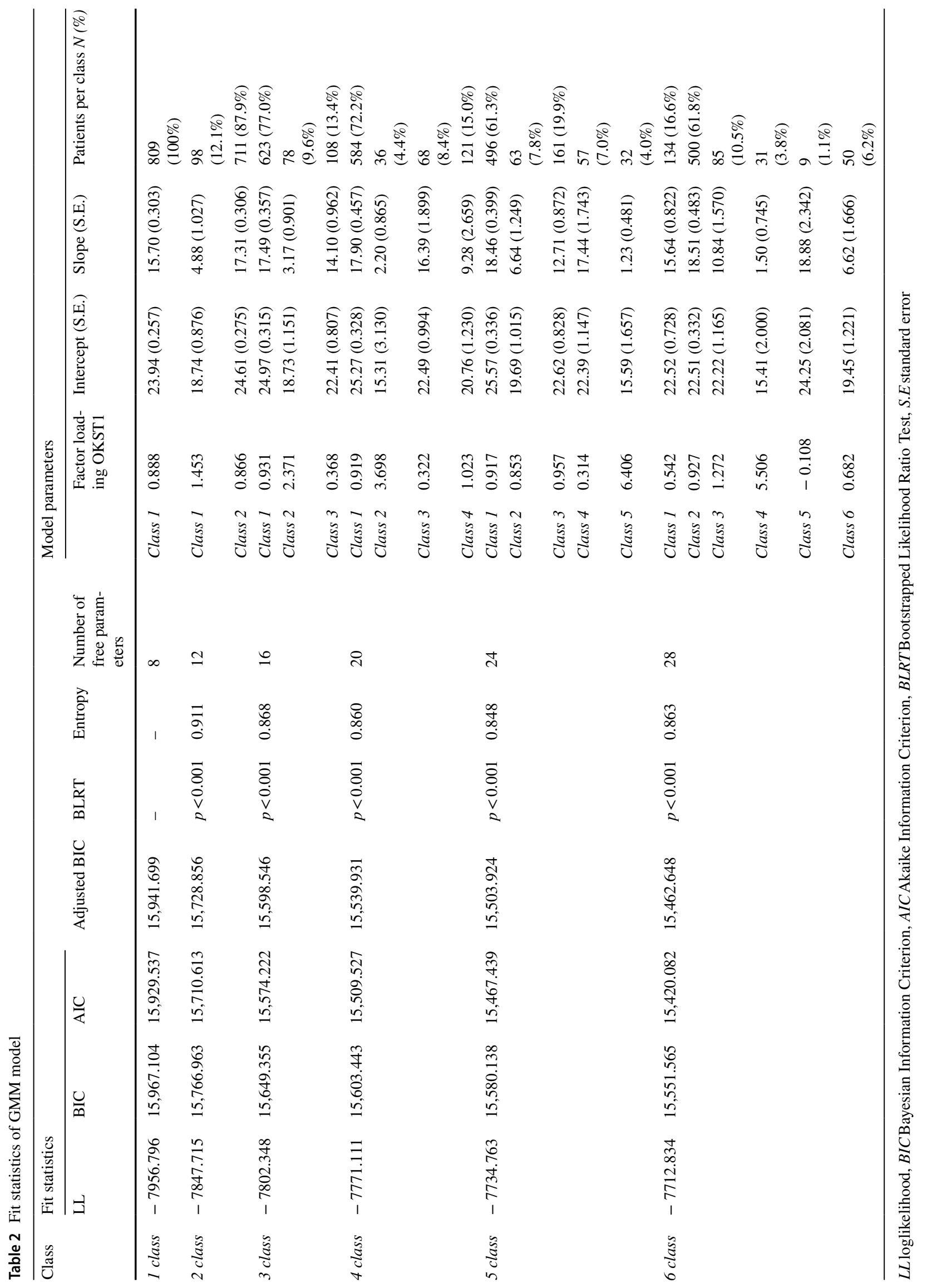




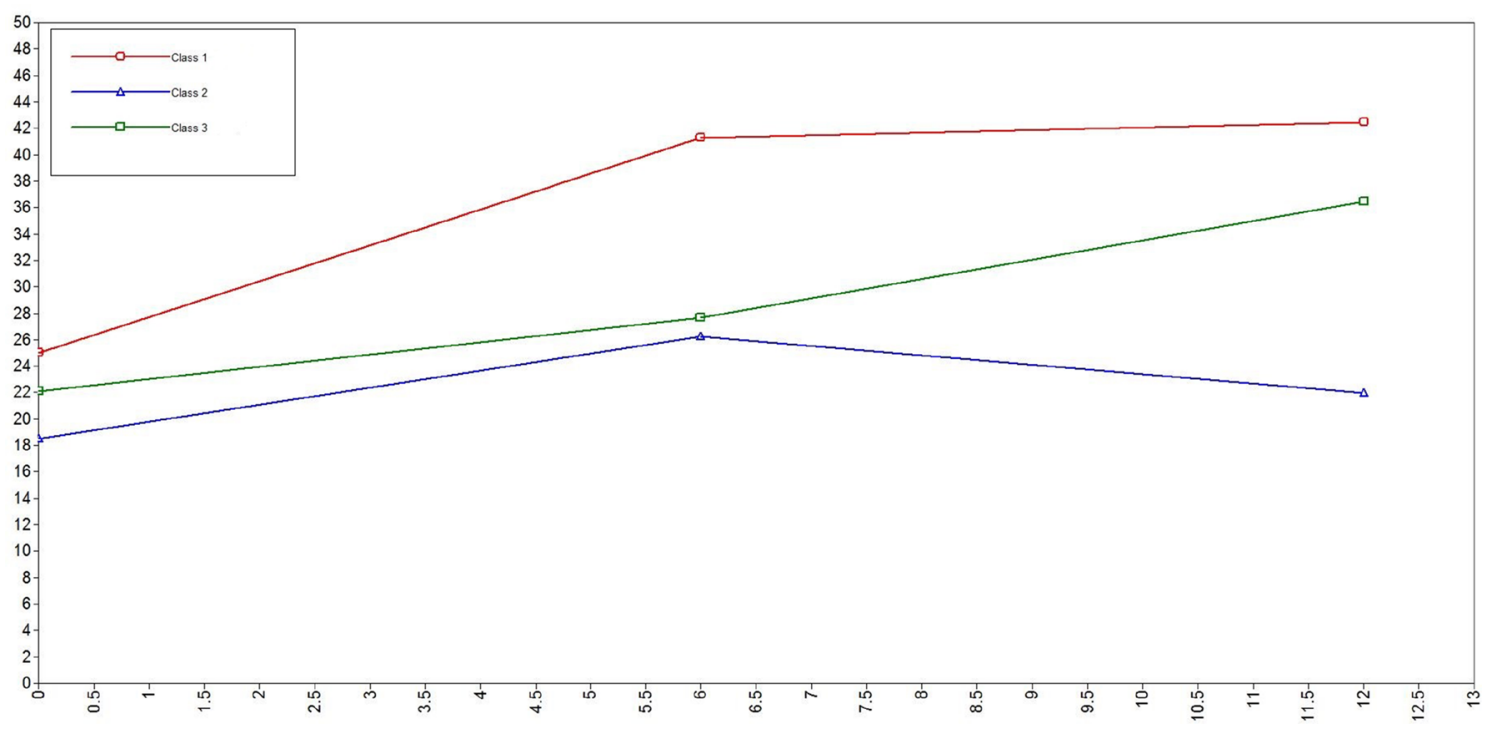

Fig. 1 Trajectories patterns of GMM 3-class model. $x$-axis: time in months, $y$-axis: OKS score. Class 1, red, 'high risers', 623 patients, $77 \%$. Class 2, green, 'gradual progressor', 108 patients, 13.4\%. Class 3, blue, 'non responder', 78 patients, $9.6 \%$

responders': ASA $\geq$ III $(2.65,1.47-4.79)$ and low scores on the EQ-5D items self-care (3.65, 2.00-6.68), anxiety/ depression $(3.43,1.96-5.98)$ and VAS health score $(0.97$, 0.96-0.99).

For the 'gradual progressors' the following variables were significant (OR, 95\% CI) for class membership: Smoking $(2.23,0.99-4.99)$ and the EQ-5D item VAS health score $(0.98,0.97-1.00)$.

\section{Multivariable analysis}

In the multivariable analysis all covariates were simultaneously entered. The largest group, 'high risers', was chosen as the reference group. Smoking was significant for the 'gradual progressors' group. The 'non responders' group was significantly associated with the EQ-5D items: selfcare, and anxiety/depression, and VAS health score, whereas ASA $\geq$ III was no longer significant in multivariable analysis. (Table 4).

\section{Discussion}

The most important finding of the present study was the identification of three subgroups with distinct recovery trajectories based on OKS after TKA. Of the subgroups, the 'high risers' could be interpreted as having the most favourable trajectory and 'non responders' as having the least favourable trajectory. Based on these present findings, as on those of previously conducted trajectory studies, patients after TKA cannot be regarded as one group and various trajectories exist.
The identification of a 'non responders' class in the present study is in line with previous studies that showed that at least one trajectory was unfavourable for pain or functional outcome [6, 7, 17].

However, another group (gradual progressors) was identified with a distinctly less favourable recovery pattern as well. Together, these two groups comprise up to $24 \%$ of the included patients. Unfortunately, this dataset did not include information on how satisfied patients were with the results of TKA. Presumably the slower recovery of the 'gradual progressors' may have been associated with less satisfaction with TKA. Future research might be able to improve the understanding of how 'gradual progressors' differ from 'non responders' and might assess the consequences for satisfaction with the outcome of TKA.

The present study showed a negative effect of psychological factors and preoperative pain scores on the outcome of TKA, which is similar to previously published studies $[14,20,25]$. In this present study, membership of a less favourable class membership (non responders or gradual progressors) was associated with worse EQ-5D scores on the items anxiety and depression. While the EQ-5D-3L has not been designed for diagnosing anxiety or depression, both anxiety and depression have been labelled as risk factors. These are potentially modifiable preoperative factors that may be used to achieve better postoperative outcome and satisfaction. This suggestion is in line with the findings of Tristaino et al. who showed that psychological support in TKA patients led to a lower incidence of anxiety and depression and faster recovery [22].

The multivariable analysis included the ASA score, the Charnley score and the EQ-VAS. These three scores could 
Fig. 2 Individual group plots for each class. High risers, gradual progressors, non responders
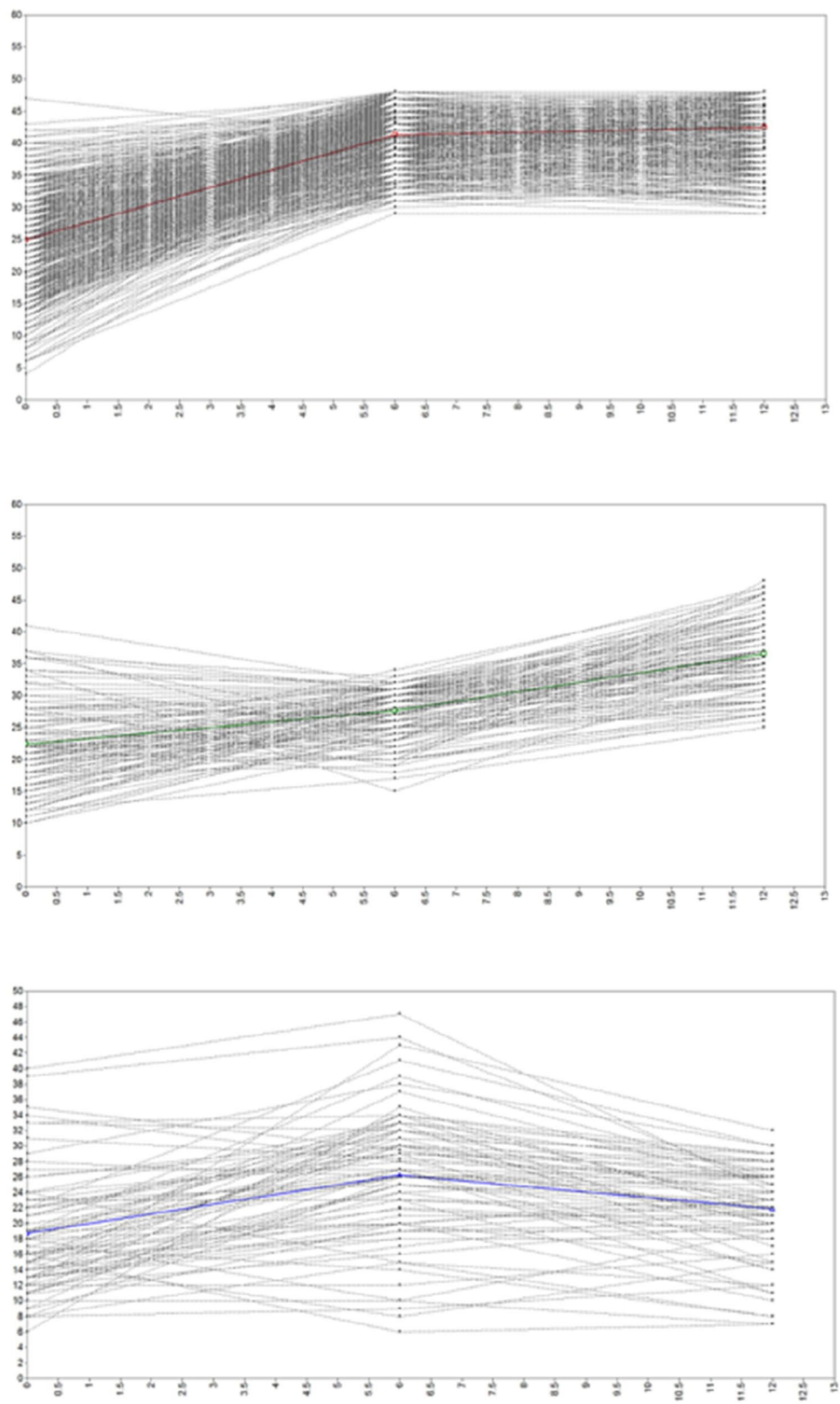

theoretically be influenced by each other and could interfere with the multivariable analysis. However, since the multivariable analysis was explorative, all three parameters were included. 
Table 3 OKS of entire sample and the three separated trajectories at the three time points

\begin{tabular}{lllll}
\hline OKS & $\begin{array}{l}\text { Entire sample } \\
(n=809)\end{array}$ & $\begin{array}{l}\text { High risers } \\
(n=623)\end{array}$ & $\begin{array}{l}\text { Gradual progressors } \\
(n=108)\end{array}$ & $\begin{array}{l}\text { Non responders } \\
(n=78)\end{array}$ \\
\hline Preoperative & $23.94(7.30)$ & $24.93(7.00)$ & $22.27(6.90)$ & $18.35(7.37)$ \\
& {$[23.44-24.44]$} & {$[24.38-25.48]$} & {$[20.95-23.58]$} & {$[16.69-20.01]$} \\
6 months postoperative & $37.89(7.90)$ & $41.25(4.47)$ & $27.01(4.04)$ & $26.05(8.40)$ \\
& {$[37.34-38.43]$} & {$[40.90-41.61]$} & {$[26.24-27.78]$} & {$[24.15-27.94]$} \\
12 months postoperative & $39.64(7.84)$ & $42.46(4.45)$ & $36.37(5.50)$ & $21.65(5.99)$ \\
& {$[39.10-40.18]$} & {$[42.11-42.81]$} & {$[35.32-37.42]$} & {$[20.30-23.00]$} \\
\hline
\end{tabular}

All OKS scores are presented as mean $( \pm \mathrm{SD})[95 \% \mathrm{CI}]$

Table 4 Multivariable analysis

\begin{tabular}{|c|c|c|c|c|}
\hline & \multicolumn{2}{|c|}{ Non responders vs high risers } & \multicolumn{2}{|c|}{ Gradual progressors vs high risers } \\
\hline & OR $(95 \% \mathrm{CI})$ & $p$ & OR $(95 \% \mathrm{CI})$ & $p$ \\
\hline Age $>75$ years vs. $\leq 75$ years & $0.97(0.44-2.16)$ & 0.941 & $0.99(0.38-2.55)$ & 0.976 \\
\hline Sex (female $)$ & $1.51(0.78-2.91)$ & 0.221 & $0.82(0.47-1.44)$ & 0.493 \\
\hline Smoking & $1.23(0.42-3.65)$ & 0.706 & $2.80(1.14-6.92)$ & 0.025 \\
\hline$A S A I I I-I V$ vs. I-II & $1.97(0.99-3.93)$ & 0.054 & $1.36(0.57-3.24)$ & 0.493 \\
\hline \multicolumn{5}{|l|}{ BMI } \\
\hline Normal weight & 1.0 & - & 1.0 & - \\
\hline Overweight (BMI 25-30) & $1.58(0.57-4.41)$ & 0.380 & $1.28(0.49-3.38)$ & 0.613 \\
\hline Obese $(\mathrm{BMI}>30)$ & $1.69(0.59-4.81)$ & 0.326 & $1.98(0.73-5.35)$ & 0.178 \\
\hline Previous surgery to the affected knee & $1.05(0.56-1.98)$ & 0.882 & $1.04(0.56-1.93)$ & 0.910 \\
\hline \multicolumn{5}{|l|}{ Charnley score } \\
\hline A & 1.0 & - & 1.0 & - \\
\hline B1 & $0.67(0.33-1.36)$ & 0.265 & $0.94(0.47-1.88)$ & 0.849 \\
\hline B2 & $1.10(0.53-2.30)$ & 0.793 & $0.86(0.38-1.94)$ & 0.721 \\
\hline $\mathrm{C}$ & $0.73(0.19-2.85)$ & 0.654 & $1.04(0.27-3.99)$ & 0.961 \\
\hline \multicolumn{5}{|l|}{$E Q-5 D$ item 'Self-Care' } \\
\hline No problems & 1.0 & - & 1.0 & - \\
\hline Some problems or unable to wash or dress & $2.22(1.09-4.54)$ & 0.029 & $1.63(0.73-3.66)$ & 0.236 \\
\hline \multicolumn{5}{|l|}{ EQ-5D item 'Usual Activities' } \\
\hline No problems & 1.0 & - & 1.0 & - \\
\hline Some problems or unable to perform usual activities & $0.92(0.35-2.42)$ & 0.858 & $1.27(0.54-2.99)$ & 0.582 \\
\hline \multicolumn{5}{|l|}{ EQ-5D item 'Pain/Discomfort' } \\
\hline No pain or discomfort & 1.0 & - & 1.0 & - \\
\hline Moderate or extreme pain or discomfort & $0.78(0.27-2.20)$ & 0.633 & $2.89(0.14-58.82)$ & 0.489 \\
\hline \multicolumn{5}{|l|}{ EQ-5D item 'Anxiety/Depression' } \\
\hline Not anxious or depressed & 1.0 & - & 1.0 & - \\
\hline Moderately or extremely anxious or depressed & $2.45(1.33-4.52)$ & 0.004 & $1.26(0.66-2.43)$ & 0.482 \\
\hline EQ-5D VAS health score (per 10 points) & $0.83(0.73-0.95)$ & 0.005 & $0.89(0.77-1.04)$ & 0.152 \\
\hline
\end{tabular}

Age was pragmatically chosen to be dichotomized at age of 75 , since literature is not clear on the age at which outcome of TKA deteriorates. However, the threshold of around 75 years has been used before in previous studies to dichotomize age groups [13].

In the present study, obesity was not found to be a risk factor for less favourable class membership. This is in line with the study of Baker et al. who found no difference in
OKS and EQ-5D between groups based on BMI [1]. These results are in contrast to the findings of Dowsey et al. [6] and Sing et al. [21], who showed obesity was a risk factor for less favourable class membership. This difference may be explained by a difference in follow-up time, since this present study investigated trajectories of the first postoperative year, whereas Dowsey et al. and Singh et al. have a follow-up of 5 years. Furthermore, this present study 
Table 5 Comparison of preoperative patient characteristics between patients with no, some and all OKS scores missing

\begin{tabular}{|c|c|c|c|}
\hline Characteristic & $\begin{array}{l}\text { No OKS scores } \\
\text { missing } \\
(N=809,1.2 \%)\end{array}$ & $\begin{array}{l}1 \text { or } 2 \text { OKS } \\
\text { scores missing } \\
(N=12.820 \text {, } \\
18.4 \%)\end{array}$ & $\begin{array}{l}\text { All OKS } \\
\text { scores miss- } \\
\text { ing } \\
(N=56.076 \text {, } \\
80.4 \%)\end{array}$ \\
\hline Age, mean (SD) & $67.2(8.1)$ & $68.4(8.7)$ & $68.7(9.1)$ \\
\hline \multicolumn{4}{|l|}{ Sex } \\
\hline Female & $63.4 \%$ & $62.4 \%$ & $64.6 \%$ \\
\hline Male & $36.6 \%$ & $37.6 \%$ & $35.4 \%$ \\
\hline \multicolumn{4}{|l|}{$B M I$} \\
\hline Underweight & $0.1 \%$ & $0.1 \%$ & $0.2 \%$ \\
\hline Normal weight & $17.7 \%$ & $16.5 \%$ & $16.4 \%$ \\
\hline Overweight & $44.1 \%$ & $41.8 \%$ & $41.1 \%$ \\
\hline Obesity & $36.5 \%$ & $38.3 \%$ & $38.4 \%$ \\
\hline Morbid obesity & $1.6 \%$ & $3.3 \%$ & $4.0 \%$ \\
\hline \multicolumn{4}{|l|}{ ASA score } \\
\hline ASA I & $13.7 \%$ & $14.3 \%$ & $14.7 \%$ \\
\hline ASA II & $69.7 \%$ & $70.0 \%$ & $69.3 \%$ \\
\hline ASA III-IV & $16.6 \%$ & $15.7 \%$ & $16.0 \%$ \\
\hline \multicolumn{4}{|l|}{ Charnley score } \\
\hline A & $49.3 \%$ & $45.3 \%$ & $42.7 \%$ \\
\hline B1 & $29.0 \%$ & $32.8 \%$ & $34.7 \%$ \\
\hline B2 & $18.6 \%$ & $18.9 \%$ & $20.0 \%$ \\
\hline $\mathrm{C}$ & $3.1 \%$ & $3.0 \%$ & $2.6 \%$ \\
\hline \multicolumn{4}{|l|}{ Smoking } \\
\hline No & $93.2 \%$ & $91.0 \%$ & $90.2 \%$ \\
\hline Yes & $6.8 \%$ & $9.0 \%$ & $9.8 \%$ \\
\hline
\end{tabular}

OKS Oxford Knee Score

used OKS, whereas Dowsey et al. used the KSS; slight differences in the content of these questionnaires may lead to differences in the effect of BMI on the outcome scores.

An unexpected and important finding is that smoking was a significant characteristic of class membership for 'gradual progressors'. This is in contrast to previously published trajectory studies, which did not consider smoking a risk factor in TKA recovery, even though it is known that worse patient-related outcomes are found in smokers [11]. Moreover, it has been shown that smoking cessation before surgery may improve postoperative outcomes [28]. Since the number of smoking patients in this present study was relatively small, however, this outcome should be interpreted with caution.

A strength of this study is the large group of patients $(n=809)$, which to the best of our knowledge is the largest so far of all studies that attempted to identify distinct trajectories after TKA. Besides, using data from a national registry with a systematic approach increased the generalizability of these findings. This is a first step in better understanding heterogeneity in recovery after TKA.

There are several limitations that need to be addressed. The limitations mainly concern the fact that this is a retrospective analysis (although all data were prospectively collected), with all its known and unknown forms of bias and has missing data (patient characteristics).

Another limitation is that patients who did not complete all three OKS questionnaires were excluded from this analysis. These patients might have not completed all three OKS questionnaires due to, for example, revision surgery during their first year. As a result, $1.2 \%$ of all primary TKA of the study period were included, which nevertheless amounted to 809 patients (Table 5). The incompleteness of OKS data can only be partially explained by the fact that a subset of the patients had revision surgery within the first year. Additionally, although the LROI started registering PROMS in 2014, not all hospitals directly started collecting PROMS immediately. Methods of collecting PROMS also vary between hospitals which might have affect completeness of OKS. Besides, highly motivated and satisfied patients are likely more motivated to complete questionnaires, whereas TKA patients with complications would have been less likely to complete further OKS, although these patients would not represent the full $98 \%$. Baseline characteristics between patients who completed OKS and those who did not or only partially completed OKS were statistically and clinically comparable. However, similarity of the groups cannot be based only on baseline patient characteristics.

A further limitation is that the intervals between the moments of completing OKS questionnaires were relatively long. Therefore, it is likely that different rehabilitation patterns and trajectories could be found if the OKS were determined more frequently or at other intervals.

The final limitation is that the LROI database does not include more detailed patient-related information, such as patient expectations, psychosocial questionnaires, and socioeconomic status. Recently Zale et al. described the importance of psychosocial factors for orthopaedic conditions [27]. Furthermore, implant properties (cruciate retaining, posterior stabilizing e.d.) were not analysed; therefore, it is still unclear if these contribute to class membership or trajectory. However, considering that Dowsey et al. [7] found no relation between type of prosthesis and class membership in a multivariable logistic model, assuming that prosthesis type has no or little influence on trajectories. 


\section{Conclusions}

Three distinct recovery patterns were found after primary unilateral TKA, namely 'high risers', 'gradual progressors' and 'non responders'. Predictors for class membership of 'non responders' are the EQ-5D items self-care and anxiety/ depression and the VAS health score. This study provides surgeons with risk factors that may help them predict which patients will face less favourable recovery trajectories.

Acknowledgements The authors would like to thank LROI for using the data. Moreover, we would like to thank the Van Rens Fonds for financially supporting this study.

Author contribution JE performed data-analysis, he wrote and revised the manuscript for important intellectual content. BH supported dataanalysis and critically reviewed the manuscript for important intellectual content. MM designed the study and critically reviewed the manuscript for important intellectual content. SV designed the study and critically reviewed the manuscript for important intellectual content and wrote the funding application. LS designed the study, provided data from the Dutch Arthroplasty Register (LROI) and critically reviewed the manuscript for important intellectual content. NM designed the study, supported data analysis and critically reviewed the manuscript for important intellectual content. JP designed the study, supported data analysis, critically reviewed the manuscript for important intellectual content and wrote the funding application. All authors approved the final version of the manuscript.

Funding This study was funded by the Van Rens Fonds Foundation (VRF2017-005), The Netherlands.

\section{Compliance with ethical standards}

Conflict of interest S.B.W. Vehmeijer has a consultancy contract with Zimmer Biomet.

Ethical approval Not applicable.

Informed consent Not applicable.

\section{References}

1. Baker P, Petheram T, Jameson S, Reed M, Gregg P, Deehan $\mathrm{D}$ (2012) The association between body mass index and the outcomes of total knee arthroplasty. J Bone Joint Surg Am 94:1501-1508

2. Baker PN, van der Meulen JH, Lewsey J, Gregg PJ, National Joint Registry for E, Wales (2007) The role of pain and function in determining patient satisfaction after total knee replacement. Data from the National Joint Registry for England and Wales. J Bone Joint Surg Br 89:893-900

3. Berlin KS, Parra GR, Williams NA (2014) An introduction to latent variable mixture modeling (part 2): longitudinal latent class growth analysis and growth mixture models. J Pediatr Psychol 39:188-203

4. Bourne RB, Chesworth BM, Davis AM, Mahomed NN, Charron KD (2010) Patient satisfaction after total knee arthroplasty: who is satisfied and who is not? Clin Orthop Relat Res 468:57-63
5. Dawson J, Fitzpatrick R, Murray D, Carr A (1998) Questionnaire on the perceptions of patients about total knee replacement. J Bone Joint Surg Br 80:63-69

6. Dowsey MM, Smith AJ, Choong PFM (2015) Latent Class Growth Analysis predicts long term pain and function trajectories in total knee arthroplasty: a study of 689 patients. Osteoarthritis Cartil 23:2141-2149

7. Dumenci L, Perera RA, Keefe FJ, Ang DC, Slover J, Jensen MP et al (2019) Model-based pain and function outcome trajectory types for patients undergoing knee arthroplasty: a secondary analysis from a randomized clinical trial. Osteoarthritis Cartil. https://doi.org/10.1016/j.joca.2019.01.004

8. Dutch Arthroplasty Register (in Dutch: LROI). Online LROIRapportage 2016: Blik op uitkomsten. https://www.lroi-rappo rtage.nl/media/pdf/PDF\%20Online\%20LROI-Rapportage\%20201 6.pdf. Accessed 9 Jan 2020

9. Dutch Arthroplasty Register (in Dutch: LROI). Online LROI annual report 2017. https://www.lroi-rapportage.nl/media/pdf/ PDF\%20LROI\%20annual\%20report\%202017.pdf. Accessed 11 Nov 2019

10. EuroQol G (1990) EuroQol-a new facility for the measurement of health-related quality of life. Health Policy 16:199-208

11. Halawi MJ, Allen DA, Baron S, Savoy L, Williams VJ, Cote MP (2018) Tobacco smoking independently predicts lower patientreported outcomes: new insights on a forgotten epidemic. $\mathrm{J}$ Arthroplast. https://doi.org/10.1016/j.arth.2018.10.036

12. Jung T, Wickrama $\mathrm{K}$ (2008) An introduction to latent class growth analysis and growth mixture modeling. Soc Personal Psychol Compass 2:302-317

13. Kuperman EF, Schweizer M, Joy P, Gu X, Fang MM (2016) The effects of advanced age on primary total knee arthroplasty: a metaanalysis and systematic review. BMC Geriatr 16:41

14. Lindberg MF, Miaskowski C, RustoEn T, Rosseland LA, Cooper BA, Lerdal A (2016) Factors that can predict pain with walking, 12 months after total knee arthroplasty. Acta Orthop 87:600-606

15. Nagin DS (2005) Group-based modeling of development. Harvard University Press, Harvard

16. Nagin DS, Odgers CL (2010) Group-based trajectory modeling in clinical research. Annu Rev Clin Psychol 6:109-138

17. Page MG, Katz J, Romero Escobar EM, Lutzky-Cohen N, Curtis $\mathrm{K}$, Fuss S et al (2015) Distinguishing problematic from nonproblematic postsurgical pain: a pain trajectory analysis after total knee arthroplasty. Pain 156:460-468

18. Rabin R, de Charro F (2001) EQ-5D: a measure of health status from the EuroQol Group. Ann Med 33:337-343

19. Ram N, Grimm KJ (2009) Growth mixture modeling: a method for identifying differences in longitudinal change among unobserved groups. Int J Behav Dev 33:565-576

20. Singh JA, Lewallen DG (2013) Medical and psychological comorbidity predicts poor pain outcomes after total knee arthroplasty. Rheumatol (Oxford) 52:916-923

21. Singh JA, O'Byrne M, Harmsen S, Lewallen D (2010) Predictors of moderate-severe functional limitation after primary total knee arthroplasty (TKA): 4701 TKAs at 2-years and 2935 TKAs at 5-years. Osteoarthritis Cartil 18:515-521

22. Tristaino V, Lantieri F, Tornago S, Gramazio M, Carriere E, Camera A (2016) Effectiveness of psychological support in patients undergoing primary total hip or knee arthroplasty: a controlled cohort study. J Orthop Traumatol 17:137-147

23. van de Schoot R, Sijbrandij M, Depaoli S, Vermunt JK (2017) The GRoLTS-checklist: guidelines for reporting on latent trajectory studies. Struct Equ Model Multidiscip J 451-467

24. van Steenbergen LN, Denissen GA, Spooren A, van Rooden SM, van Oosterhout FJ, Morrenhof JW et al (2015) More than 95\% completeness of reported procedures in the population-based Dutch Arthroplasty Register. Acta Orthop 86:498-505 
25. Vissers MM, Bussmann JB, Verhaar JA, Busschbach JJ, BiermaZeinstra SM, Reijman M (2012) Psychological factors affecting the outcome of total hip and knee arthroplasty: a systematic review. Semin Arthritis Rheum 41:576-588

26. Wickrama KAS, Lee TK (2016) Higher-order growth curves and mixture modeling with mplus: a practical guide.

27. Zale EL, Ring D, Vranceanu AM (2018) The future of orthopaedic care: promoting psychosocial resiliency in orthopaedic surgical practices. J Bone Joint Surg Am 100:e89

28. Zhao S, Chen F, Wang D, Wang H, Han W, Zhang Y (2019) Effect of preoperative smoking cessation on postoperative pain outcomes in elderly patients with high nicotine dependence. Medicine (Baltimore) 98:e14209

Publisher's Note Springer Nature remains neutral with regard to jurisdictional claims in published maps and institutional affiliations. 